\title{
MEDICAL ALERT SYSTEM FOR REMOTE HEALTH MONITORING USING SENSORS AND CLOUD COMPUTING
}

\author{
Indumathy $\mathbf{N}^{1}$, Dr.Kiran Kumari Patil ${ }^{2}$ \\ ${ }^{1}$ M Tech Scholar, Dept of CSE, Reva Institute of Technology and Management, Bangalore, India. \\ ${ }^{2}$ Associate Professor, Dept of CSE, Reva Institute of Technology and Management, Bangalore, India
}

\begin{abstract}
Remote monitoring of patient's health and generating alert in case of emergency situations is an important aspect in healthcare. Sensors play an important role in this regard. They measure the physical parameters and provide real-time data but sensors are constrained with limited battery, storage and processing. Cloud has several advantages such as huge memory, high bandwidth, faster data processing, on-demand service. Therefore we integrate sensors with Cloud computing to propose a system that keeps track of patient's health in a timely manner and generates voice alert when the patient's vital parameters crosses the normal value. The measured data is sent to Cloud from where doctors can access it via Internet.
\end{abstract}

Key Words: Wireless sensors, Cloud computing, Health monitoring, Alert messages

$* * *$

\section{INTRODUCTION}

Wireless sensor network (WSN) typically consists of a collection of sensors with their own power supply, wireless communication, data storage, and data processing capability. In a typical sensor network, each sensor node has a microprocessor with limited processing capability, a small amount of memory for signal processing and has limited energy supply and bandwidth. Each sensor node communicates wirelessly with a few other local nodes within its radio communication range. Information collected by and transmitted on a sensor network describes conditions of physical environments-for example, temperature, humidity, or vibration. Applications of sensor networks are wide ranging and can vary significantly in application requirements. Sample applications include: Traffic monitoring, Habitat monitoring, Forest fire detection, Natural disaster prevention, Healthcare monitoring etc.

Cloud computing is the use of computing resources (hardware and software) that are delivered as a service over the network (mainly the Internet). Cloud has abundant processing power, large amount of storage which can be scaled according to application needs. Modern technology is being shifted to Cloud based platform as it is suited for longterm data storage.

The proposed system focuses on collection of patient's vital health parameters and generates alert to care takers, doctors so that immediate action can be taken in case of emergencies. The data is then stored in Cloud so that data can be accessed via Internet from anywhere anytime.

Sensors are used to collect health data from patients. Instead of attaching sensors to medical equipments, wearable sensors are attached to human body which continuously monitor the blood pressure rate, heartbeat, fall detection, etc. These sensor nodes help in continuous health monitoring of post operative patients at hospital and elderly patients at home environment with almost real time updates of medical records. The data are published via web servers. Healthcare professionals, researchers and patients can access the longterm physiological data via the Internet. A secure cloud server allows authenticated users to access real-time patient information

\section{LITERATURE SURVEY}

Researchers at MIT lab have developed MIThril, a wearable computing platform compatible with both custom \& off the shelf sensors. This paper "Context- aware Human Activity Recognition and Decision Making" proposes a project called "code blue" which is based on developing WSN for medical applications. When sensors are attached to human body, it transmits vital signals by the help of ad-hoc network to healthcare providers. A voice enhanced service using voice over internet protocol (VoIP) is provided in order to communicate with the patients for better diagnosis of their problems and to provide better aid. Once the data from sensor nodes are collected they are then uploaded to the cloud gateway, the gateway classifies data into health data, gyroscope, accelerometer data, imaging data and finally store in a local database.

Much work is being performed to integrate services of WSN and cloud computing. But this work is only in initial stages and facing challenges of the real world. Some of the real world challenges in the area of Wireless sensors networks are summarized here. WSNs have been deployed in various application domains, including health care system that monitor human health and provide life care services. Current systems are based on the central server i.e. all the monitoring and respective services are stored and processed centrally. This leads to couple of high risk factors associated with it such as low performance, prone to failure, high 
maintenance cost and limited services. This paper "A Survey on Wearable Sensor-Based Systems for Health Monitoring and Prognosis" proposes a Sensor cloud technology enables the early detection of adverse conditions and diseases. A number of recent research focuses on wearable sensors which are attached to patient's body to regulate their health condition. A secure cloud server allows authenticated users to access real-time patient information to consult with medical specialists located at distant places.

\section{SYSTEM ARCHITECTURE}

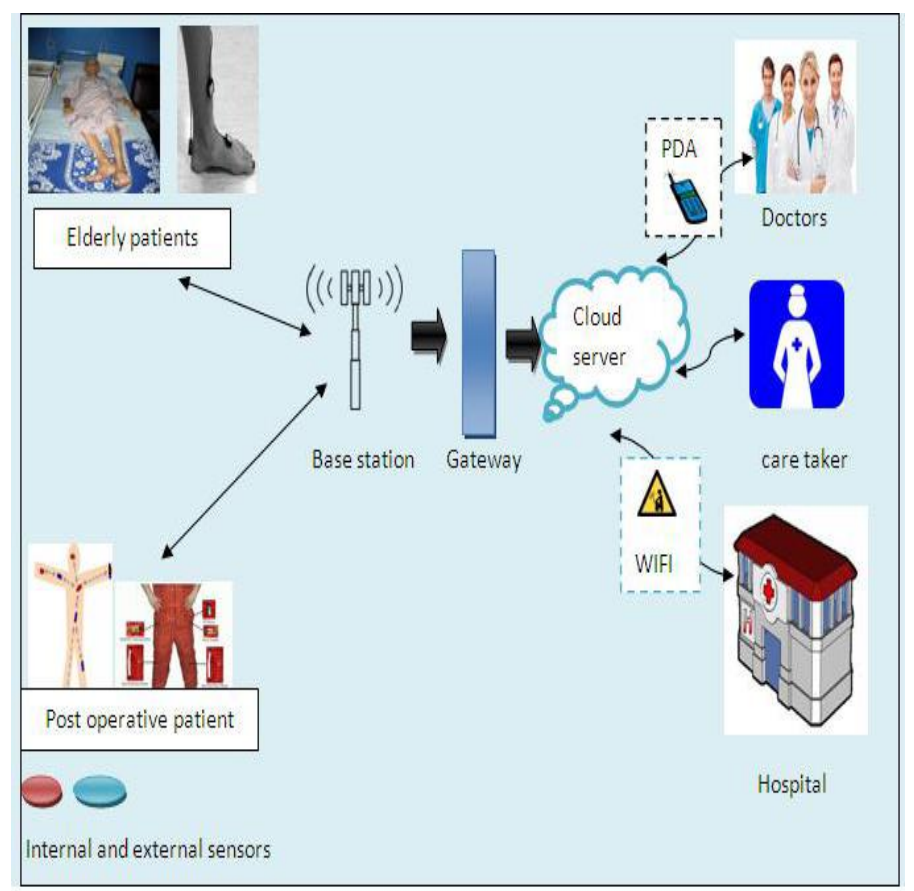

Fig 1: Patient monitoring and alerting system

In recent times, most homes moved to smart home which contains sensor appliances like smart computers, TVs, smart floors which contain sensor nodes in it to detect human activities. A wireless system could be set up throughout the home to sense physiological parameters such as ECG, heart rate, body temperature, etc., motion patterns and visual detection of elderly patients.

This system consists of the front end sensors, patient station, and Cloud server. The front end is composed of different types of sensors for monitoring continuous physiological parameters. We have used heart rate sensors and temperature sensors for physiological monitoring. To extend the system in order to monitor the motion patterns and visual detection of elderly patients, accelerometer sensors can be attached to human arm to determine patients' movements and 3D cameras can be used for visual monitoring of elderly patients. Sensor nodes are responsible for sensing and sending the signals. The received signal is sent to android application where the received values are checked against threshold values. When an anomaly is detected, the application generates an alert to the doctors and caretakers via a Personal Data Assistant (PDA) or cell phones. The above architecture shows how sensor cloud serves different group of patients.

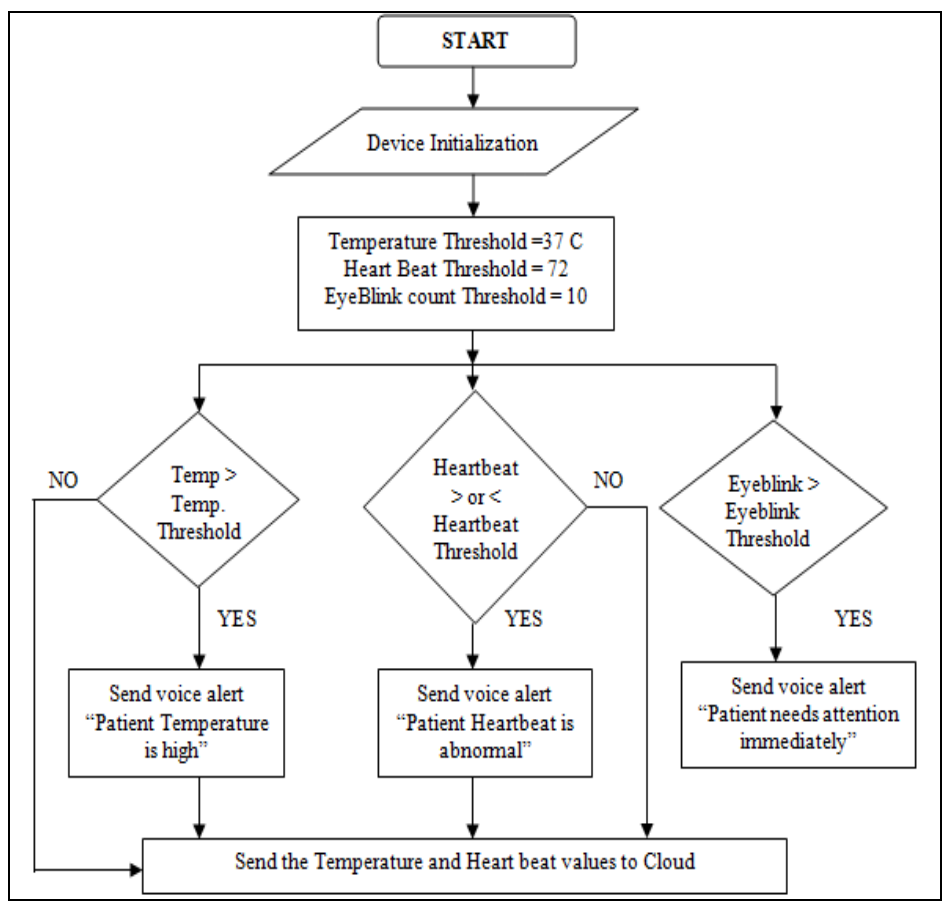

Fig 2: Flowchart showing the flow of events in the system

The sensors collect the patient vital parameters such as temperature and blood pressure. Eye blink sensor is used for post operative patients or stroke patients who wants somebody's help immediately but cannot walk or lift hands or speak to draw attention of doctors or care takers. In such cases, the patient can blink his/her eye which is detected by eye blink sensor. For example if the threshold is set as 5, and if the patient blinks the eye 5 times, a voice alert is sent to the doctor's mobile number which is pre-programmed in the hardware program (Embedded $\mathrm{C}$ program loaded into IC). Similarly for temperature and pressure, threshold value is set which is compared every time with the measured value and a voice alert is sent if measured value is greater than threshold. All the sensed values are sent to the Cloud server where the data is stored for long-term analysis and interpretation. Doctors can access the data in the Cloud via Internet. 


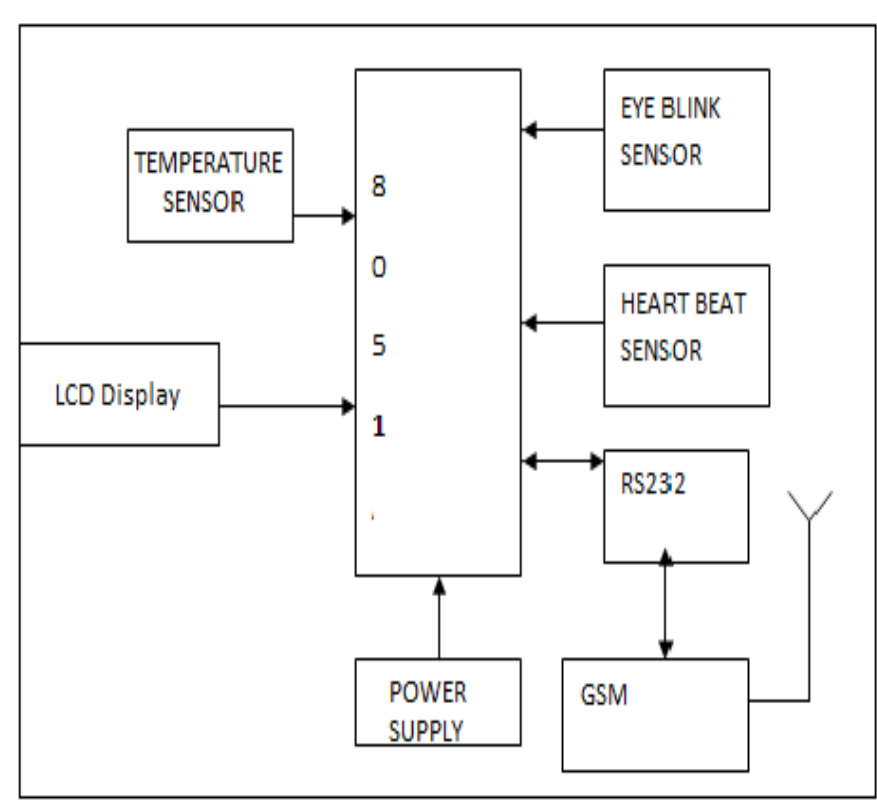

Fig 3: Hardware architecture of the proposed system

The Hardware part consists of Temperature sensor (LM35), Heart beat sensor and Eye blink sensor, Power supply unit, GSM unit (SIM 300), 8051 development board, P89V51RD2 IC, LCD and ADC card (0809). ADC0809 is an 8-bit analog to digital converter. It is used to convert the analog voltage of temperature sensor and battery circuit. The reference voltage of ADC0809 is $5 \mathrm{~V}$. It is an 8 channel ADC. The temperature sensor is connected to channel 0 and battery circuit is connected to channel 1 . Keil software provides the ease of writing the code in C, U-VISION 2 the new IDE from Keil Software combines Project management, Source Code Editing and Program Debugging in one powerful environment. It acts as a cross-compiler.

The main centre part of the project is the microcontroller. Here we are using the 8051 based Philips P89V51RD2 microcontroller. The P89V51RD2 are 80C51 microcontrollers with $64 \mathrm{kB}$ flash and $1024 \mathrm{~B}$ of data RAM. The microcontroller is programmed in Embedded $\mathrm{C}$ and compiled using KEIL software to generate HEX file which is loaded into the IC using Flash magic software.

The mobile numbers of doctor and caretaker are hardcoded in the Embedded $\mathrm{C}$ code. The Microcontroller is programmed such that it sends the keyword as TEMP for temperature value along with the value measured by temperature sensor as sms through the GSM unit (Ex. TEMP $=38 C$ ). Similarly HR (heart rate) is used as keyword corresponding to Heart beat rate and the value of heart rate measured by Heart beat sensor $(E x . H R=76)$. The keyword corresponding to Eye blink is EB. $(\mathrm{Ex} . \mathrm{EB}=8)$

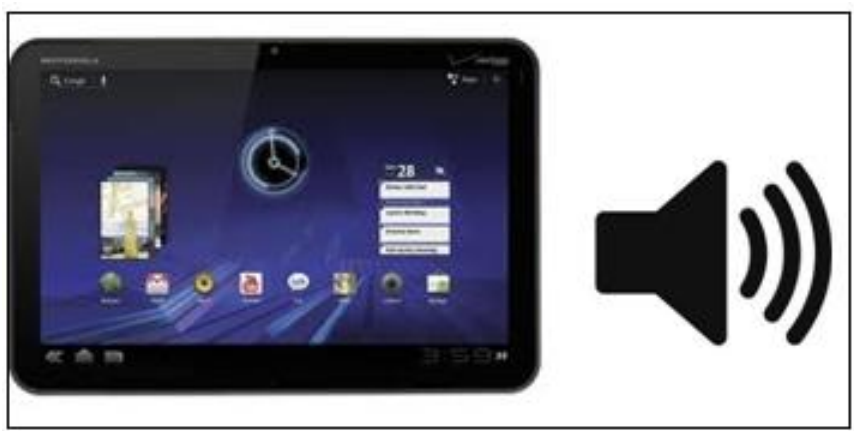

Fig 4: Android application

Once the Android (receiver) receives the sms, the keyword (TEMP or HR or EB) is matched in the android application where the threshold values for temperature, heart beat and eye blink are programmed. The value received in sms is compared with the threshold value by the android application, if the value is equal to threshold; it is stored in SQLite database in the column corresponding to temperature for the respective patient ID. If the value is greater than threshold the keyword is matched to a text and sent to TTS (text-to-speech) engine which speaks out the text to generate voice alert saying "Patient temperature is high", then the value is stored in database. Similar is the workflow for Heart beat and Eye blink operations. But for Eye blink operation we are not storing the value in database as it has nothing to interpret. The idea of using Eye blink sensor is to help patient to draw the attention of caretaker immediately in case of emergencies as a voice alert is generated in doctor and caretaker's mobile when the Eye blink value crosses the threshold.

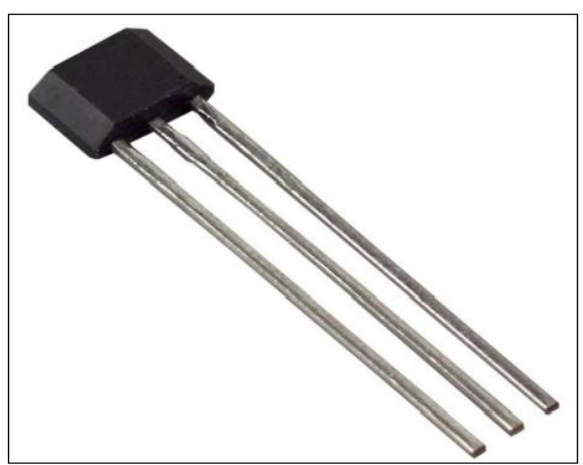

Fig 5: Temperature sensor

Function: It is calibrated in Celsius, suitable for remote applications. LM35 operates from 4 to 30 volts. Its main application is detection of heat, so it is used as temperature sensor. 


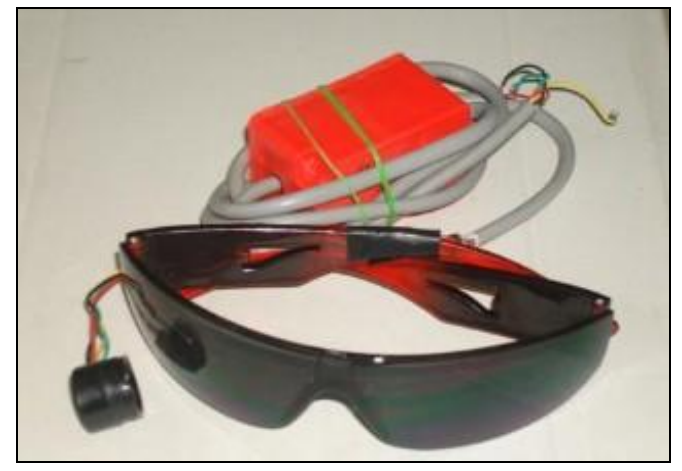

Fig 6: Eye Blink sensor

Function: The Eye Blink sensor is IR based. The Variation across the eye will vary as per eye blink. If the eye is closed means the output is high otherwise output is low. This is to know whether the eye is in closing or opening position. This output is given to logic circuit to indicate the alarm.

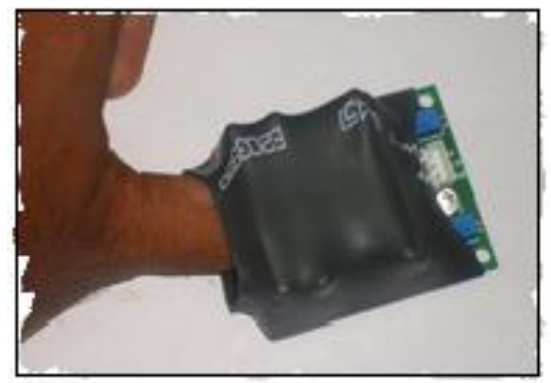

Fig 7: Heart beat sensor

Function: The Heart Beat Sensor provides a simple way to study the heart's function. This sensor monitors the flow of blood through Finger. As the heart forces blood through the blood vessels in the Finger, the amount of blood in the Finger changes with time.

\section{IMPLEMENTATION}

The project implementation mainly consists of three modules: Hardware, Android application and Cloud. Hardware module is responsible for measuring patient's vital parameters such as temperature, heart rate and sensing eye blink values, sending these values along with the respective keywords in sms to the hardcoded mobile numbers. Android application is responsible for detecting abnormality and generating voice alerts, and to store the values in SQLite database. The data is then stored in the Cloud and published via Web servers so that doctors and care takers can access the data via Internet. HttpPost request is used to send data from SQLite DB to a php page on the web server which will be stored in mysql DB. A front end page is created using html where doctors can login to see patient information which is retrieved from mysql DB through php.

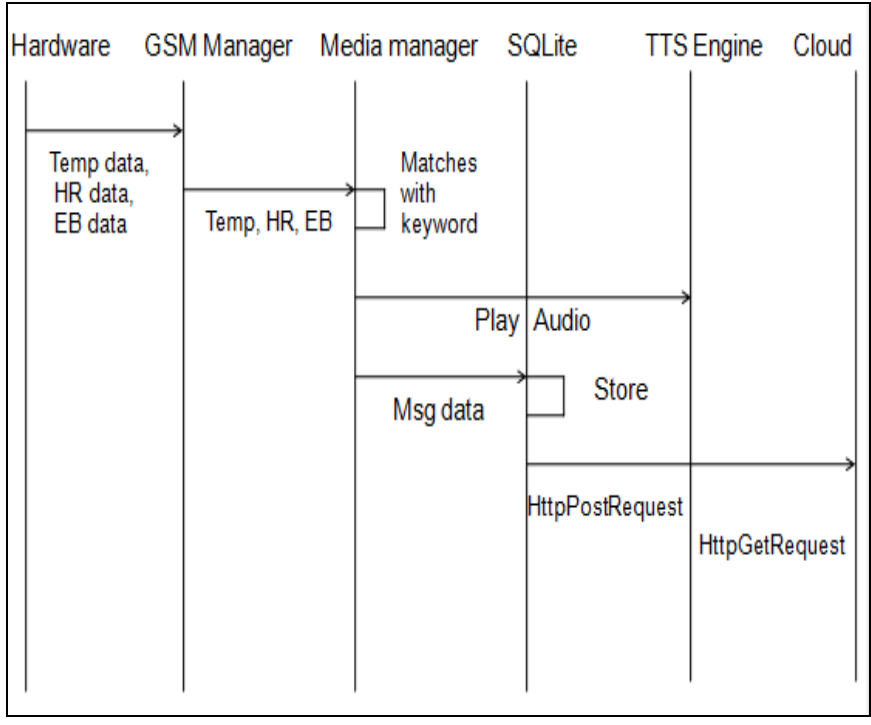

Fig 8: Sequence diagram showing the interactions in the system

Hardware -- consists of Temperature sensor, Heart beat sensor, Eye blink sensor, GSM unit, Power supply, 8051 microcontroller. The GSM unit sends the value measured by the sensor as a message to a given phone number

GSM manager -- This is a free version of android app. It can be remotely controlled by sending sms to the phone number. GSM manager receives the message sent by the GSM unit in hardware

SQLite - SQLite is a software library that is selfcontained, serverless, zero-configuration transactional SQL database engine. Temperature and heart beat values are stored in SQLite database

TTS Engine -- Text-To-Speech (TTS) enables the Android device to "speak" text. It generates the voice alert when temperature or pressure value is above threshold and also when patient needs attention.

Cloud -- A remote computer is configured as web server using php and mysql. Data from SQLite DB is sent to this remote server through Http Post/Get requests. Users can access the remote server (Cloud) to obtain patient's data.

\section{CONCLUSION}

The applications of Wireless Sensor Networks and Cloud computing in the field of Healthcare are discussed in this paper. Disadvantages of WSN are compensated by the advantages offered by Cloud computing, so both these technologies are combined in the proposed system.

Sensors enabled patient monitoring system using cloud computing monitors patient health and shares information among doctors, care-takers. Future enhancements would be to increase Cloud security as the data being stored is 
Sensitive health-related data. Security mechanisms such as authorization, authentication and user access control must be incorporated in the system. Different policy management should be implemented in order to enhance the privacy and security.

\section{REFERENCES}

[1] Subasish Mohapatra, K.Smruti Rekha, "Sensor-Cloud: A Hybrid Framework for Remote Patient Monitoring". International Journal of Computer Applications (09758887). Volume 55- No.2, October 2012.

[2] Sajjad Hussain Shah, Assad Iqbal, Syed Shaukat Ali Shah, "Remote Health Monitoring through an Integration of Wireless Sensor Networks, Mobile Phones \& Cloud Computing Technologies". IEEE 2013 Global Humanitarian Technology Conference.

[3] Asad Masood Khattak, La The Vinh, Dang Viet Hung, Phan Tran Ho Truc, Le Xuan Hung, D. Guan, Zeeshan Pervez, Manhyung Han, Sungyoung Lee, and Young-Koo Lee, "Context- aware Human Activity Recognition and Decision Making”, IEEE HealthCom, Korea, 2010, PP. 113117.

[4] Alexandros Pantelopoulos and Nikolaos G. Bourbakis, "A Survey on Wearable Sensor-Based Systems for Health Monitoring and Prognosis", IEEE Trans. on Systems, Man, and Cybernetics- PART C: Applications and Reviews, USA, Volume- 40, No- 1, January 2010, PP. 4- 10.

\section{BIOGRAPHIES}

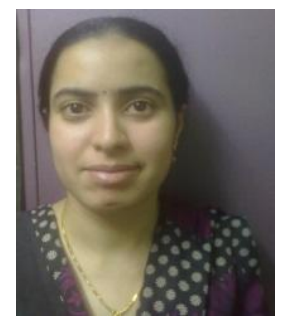

Indumathy completed B.E in Computer Science Engineering from SJCIT, pursuing MTech in Computer Network Engineering at RITM, Bangalore. Interested in the field of Networks, SAN, and Cloud Computing.

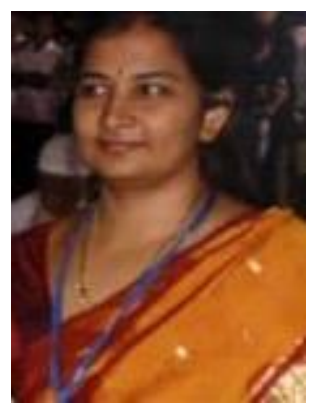

Dr. Kiran Kumari Patil has pursued M.S in Germany and Ph.D in Computer Networks. Works as Associate Professor at Reva Institute of Technology and Management and she is the PG Co-ordinator for MTech CNE at RITM, Bangalore. 